\section{Erythrodermic psoriasis treated with apremilast}

\author{
John Arcilla, ${ }^{1}$ Daniel Joe, ${ }^{1}$ \\ Johnathan Kim, ${ }^{1}$ Yohanan Kim, ${ }^{1}$ \\ VuAnh N. Truong, ${ }^{1}$ Navin Jaipaul ${ }^{1,2}$ \\ 'Department of Medicine, Loma Linda \\ University School of Medicine and \\ 2Department of Medicine, VA Loma Linda \\ Healthcare System, Loma Linda, CA, USA
}

\begin{abstract}
Erythroderma is a rare potentially deadly exfoliative dermatitis characterized by diffuse cutaneous erythema which may be associated with multi-organ dysfunction. Therefore, it is imperative to recognize and treat it promptly. Erythrodermic psoriasis is the most common form of erythroderma. Management of this condition is largely based on aggressive supportive care and the use of anti-inflammatory immunosuppressive and biologic agents. We describe a case of psoriatic erythroderma which was triggered by withdrawal from systemic steroids and successfully treated with apremilast and cyclosporine. Apremilast induced atrial fibrillation limited its continued use after the initial response period.
\end{abstract}

\section{Introduction}

We report a case of a 79 year old man with erythrodermic psoriasis successfully treated during the initial response phase with the novel oral small-molecule phosphodiesterase-4 inhibitor apremilast.

\section{Case Report}

A 79-year-old man with hypertension and psoriasis was hospitalized for severe sepsis associated with a generalized and painful erythematous rash. He had been diagnosed with psoriasis affecting $<1 \%$ body surface area (BSA) three months before this presentation and was treated with topical ketoconazole and fluocinolone. Two weeks before hospitalization, he received a five day oral prednisone taper prescribed by a family physician for skin rash. Following this, he developed a confluent, erythematous, scaling rash covering $>50 \%$ BSA. Methotrexate was started for presumed psoriatic erythroderma; however, his symptoms worsened to include progressive skin involvement, fever, and hypotension which led to hospitalization.Physical exam revealed tender erythematous plaque with scale from head to toe, most prominently involving the head, neck, chest, back, upper arms, abdomen, buttocks, groin, and proximal thighs (Figure 1). Laboratory evaluation was remarkable for leukocytosis of $23.8 \times 103 / \mu \mathrm{L}$ and pre-renalazotemia. Intravenous fluids, empiric antibiotics, topical steroids, and emollient moisturizer were started. Infectious work-up was negative; yet fevers, leukocytosis, and cutaneous pain symptoms persisted. Dermatology performed a punch biopsy which demonstrated evolving pustular psoriasis (Figure 2). Apremilast was started for treatment of erythrodermic psoriasis. The patient's rash and systemic features began to improve by day 10 . Though BSA involvement was essentially
Correspondence: Navin Jaipaul, VA Loma Linda Healthcare System, 11201 Benton St. 111N, Loma Linda, CA 92357, USA

Tel.: +1.909.583.6090 - Fax: +1.909.777.3858.

E-mail: Navin.Jaipaul@va.gov

Key words: Erythroderma; Psoriasis; Erythrodermic psoriasis; Apremilast.

Contributions: the authors contributed equally.

Conflict of interest: the authors declare no potential conflict of interest.

Received for publication: 13 May 2016.

Accepted for publication: 17 August 2016.

This work is licensed under a Creative Commons Attribution-NonCommercial 4.0 International License (CC BY-NC 4.0).

(C) Copyright J. Arcilla et al., 2016

Licensee PAGEPress, Italy

Dermatology Reports 2016; 8:6599

doi:10.4081/dr.2016.6599

unchanged, psoriasis plaques demonstrated reduced erythema and scaling with marked improvement in cutaneous pain symptoms (Figure 3). As a result, Psoriasis Area and Severity Index score improved from 44.0 on admission to 26.4 after initiating apremilast treatment. However, the patient subsequently developed new-onset atrial fibrillation attributed to apremilast which was discontinued and switched to cyclosporine. The patient continued to improve and was discharged on cyclosporine.
A)

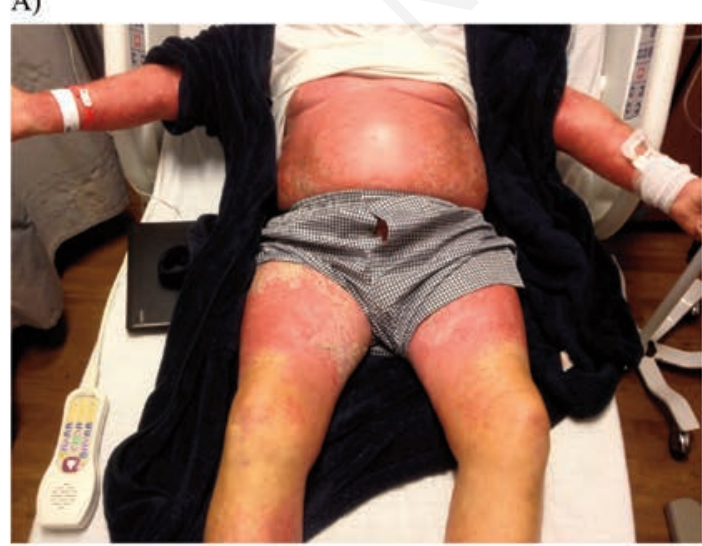

B)

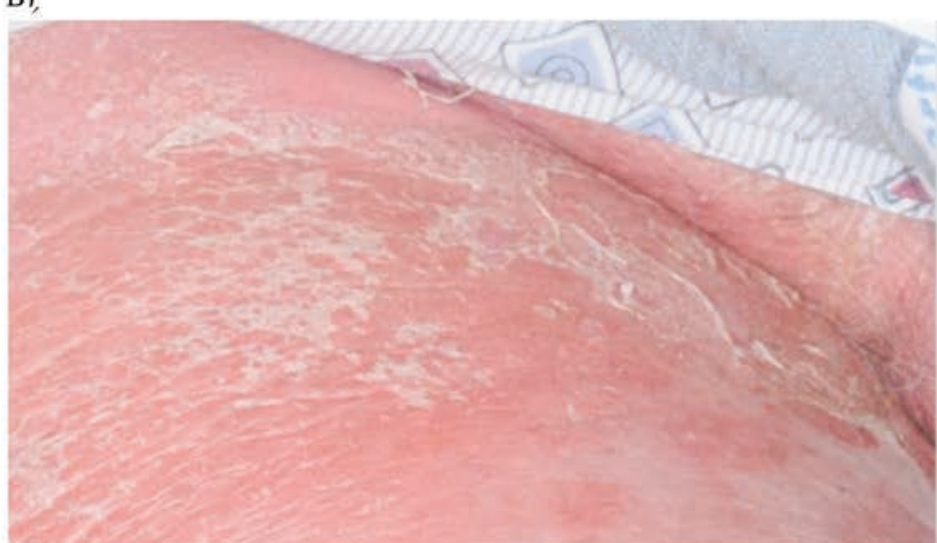

Figure 1. Skin findings on day one of hospitalization demonstrating (A) well defined generalized erythema and scaling most prominently involving the head, neck, chest, back, upper arms, abdomen, buttocks, groin, and proximal thighs and (B) magnified view of the erythema and scaling. 


\section{Discussion}

Erythroderma is a rare potentially deadly exfoliative dermatitis which may result from systemic infection, drug hypersensitivity, malignancy (e.g. cutaneous T cell lymphoma), and inflammatory conditions such as Stevens Johnson syndrome, pityriasis rubra pilaris, and erythrodermic psoriasis. ${ }^{1}$ Initial management for severe cases includes fluid resuscitation, empiric antibiotics, and diligent diagnostic evaluation.

Erythrodermic psoriasis is the most common form of erythroderma and accounts for $25 \%$ of all cases. ${ }^{2}$ When associated with psoriasis, the erythrodermic variant represents less than $1.5 \%$ of cases and manifests with welldefined erythematous plaques and overlying silvery scale. ${ }^{3}$ It generally affects the entire body and may be associated with life-threatening complications such as sepsis, hypovolemic shock, and acute kidney injury secondary to cutaneous fluid loss. ${ }^{2}$ Erythrodermic psoriasis may result from uncontrolled dermatosis, abrupt withdrawal of anti-psoriatic drugs such as steroids or methotrexate, drug reaction, systemic infection, ultraviolet burns, alcoholism, and emotional stress. ${ }^{2}$ Our patient's withdrawal from oral steroids likely triggered his erythroderma. Skin biopsy may be helpful in diagnosis as erythroderma may develop acutely or gradually from any variant of psoriasis. ${ }^{2}$ Treatment is largely based on aggressive supportive care and the use of anti-inflammatory immunosuppressive and biologic agents. High quality evidence-based treatment recommendations for erythordermic psoriasis are lacking due to the rarity of the condition. Most randomized clinical trials on psoriasis treatments have excluded less common variants such as the erythrodermic and pustular subtypes. ${ }^{4}$ In light of this, first-line agents that have been used with variable efficacy include cyclosporine, infliximab, acitretin, and methotrexate. ${ }^{5}$ Etanercept and combination therapies are second-line alternatives. ${ }^{5}$ Recently, biologics including ustekinumab and golimumab have also been used with reported efficacy in the treatment of erythrodermic psoriasis. ${ }^{6,7}$ Systemic corticosteroids and ultraviolet light therapy are not recommended due to risks of disease exacerbation and photosensitivity. ${ }^{5}$ Choice of therapy should be based on disease severity and patient comorbidities. For example, we chose to treat our patient with apremilast due to the presence of pre-renal acute kidney injury which is a relative contraindication to use of the other aforementioned first-line agents. Apremilast is a novel small-molecule oral medication which selectively inhibits phosphodiesterase 4 and has been shown to downregulate the production of pro-inflammatory cytokines while upregulat- ing anti-inflammatory cytokines. ${ }^{8}$ It is approved for treatment of psoriatic arthritris and moderate to severe plaque psoriasis. The efficacy of apremilast was demonstrated in a phase III randomized controlled trial that showed a statistically and clinically significant

reduction versus placebo in the baseline Psoriasis Area and Severity Index score in patients with moderate to severe plaque psoriasis. ${ }^{9}$ However, a search of the published literature resulted in no reports describing the use of apremilast in erythrodermic psoriasis. Our

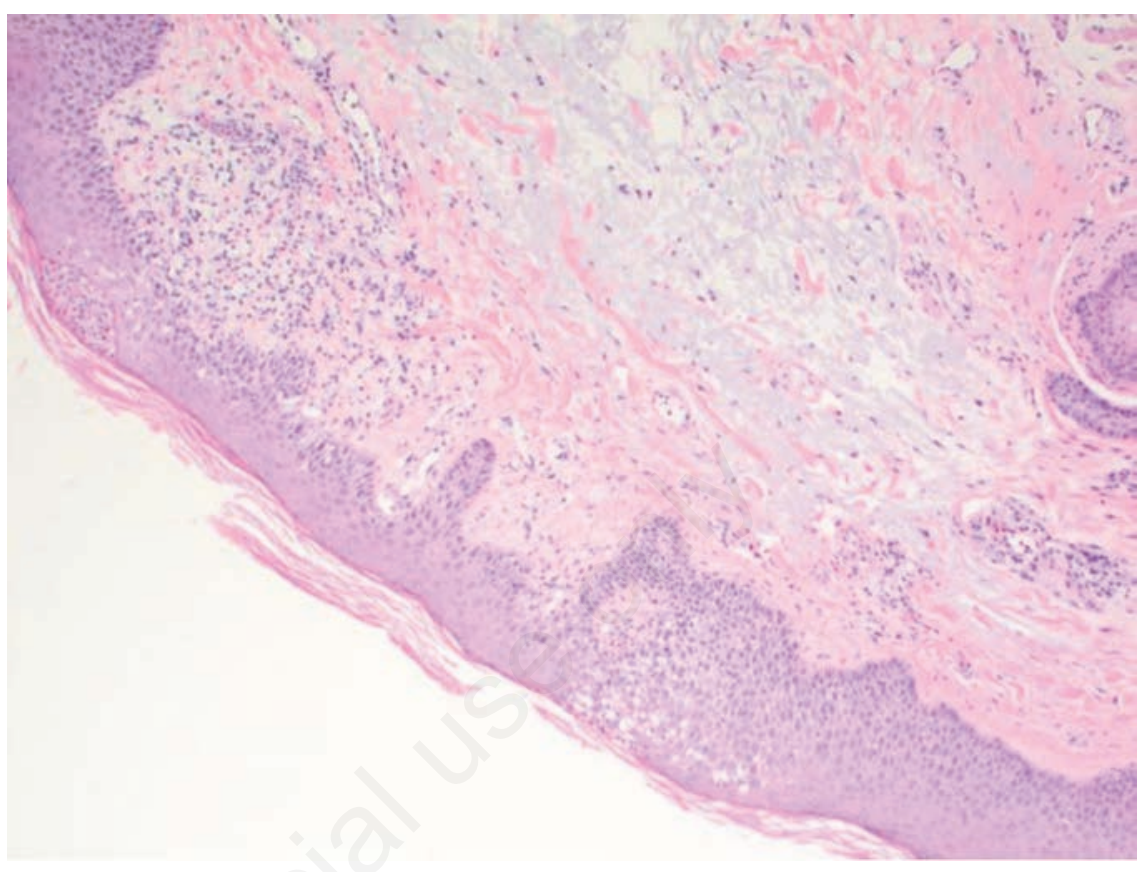

Figure 2. Skin biopsy demonstrating mild psoriasiform hyperplasia and rare intracorneal pustules with mild superficial perivascular mixed inflammation consistent with evolving pustular psoriasis.

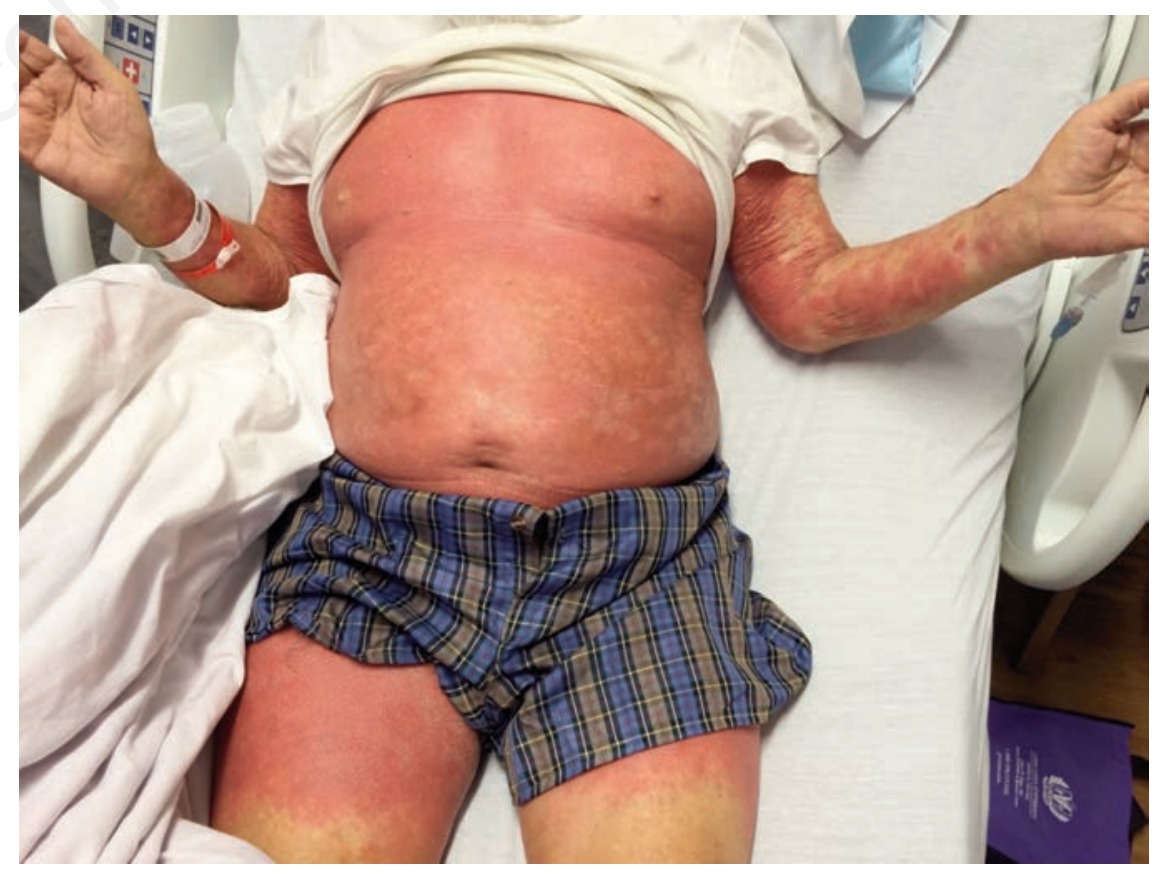

Figure 3. Skin findings on day ten of hospitalization demonstrating reduced intensity of erythema and scaling in response to apremilast treatment. 
patient improved on apremilast; however, he developed new-onset atrial fibrillation which was attributed to the medication so it was discontinued. When compared with placebo, apremilast treatment has been associated with an increased, albeit low, incidence of tachyarrhythmia, most frequently atrial fibrillation..$^{9}$ Once the patient's renal function improved, he was started on cyclosporine and his erythroderma eventually resolved.

\section{Conclusions}

Erythroderma associated with psoriasis may be triggered by withdrawal from systemic steroids. It is a rare and potentially fatal exfoliative dermatitis which needs to be recognized and treated promptly. The mainstay of treatment is founded on aggressive supportive care and use of anti-inflammatory immunosuppressive drugs, including newer biologic agents. We also observed encouraging results in treating erythrodermic psoriasis using the novel oral small-molecule phosphodiesterase-4 inhibitor apremilast. Its continued use in our patient, however, was limited after the initial response period by the development of atrial fibrillation which was attributed to the medication.

\section{References}

1. Rothe MJ, Bernstein ML, Grant-Kels JM. Life-threatening erythroderma: diagnosing and treating the red man. Clin Dermatol 2005;23:206-17.

2. Stinco G, Errichetti E. Erythrodermic psoriasis: current and future role of biologicals. Bio Drugs 2015;29:91-101.

3. Raychaudhuri SK, Maverakis E, Raychaudhuri SP. Diagnosis and classification of psoriasis. Autoimmun Rev 2014;13:490-5.

4. Menter A, Korman NJ, Elmets CA, et al. Guidelines of care for the management of psoriasis and psoriatic arthritis: sections $1,3,4,5$. Overview of psoriasis and guidelines of care for the treatment of psoriasis with biologics. J Am Acad Dermatol 2008;58:826-50.
5. Rosenbach M, Hsu S, Korman NJ, et al. Treatment of erythrodermic psoriasis: from the medical board of the National Psoriasis Foundation. J Am Acad Dermatol 2010;62:655-62.

6. Kim YS, Kim JH, Lee $\mathrm{S}$, et al. Erythrodermic psoriasis improved by ustekinumab: a report of two cases. Ann Dermatol 2016;28:121-2.

7. Lee WK, Kim GW, Cho HH, et al. Erythrodermic psoriasis treated with golimumab: a case report. Ann Dermatol 2015;27:446-9.

8. Kelly JB, Foley P, Strober BE. Current and future oral systemic therapies for psoriasis. Dermatol Clin 2015;33:91-109.

9. Papp K, Reich K, Leonardi CL, et al. Apremilast, an oral phosphodiesterase 4 (PDE4) inhibitor, in patients with moderate to severe plaque psoriasis: results of a phase III, randomized, controlled trial (Efficacy and Safety Trial Evaluating the Effects of Apremilast in Psoriasis [ESTEEM] 1). J Am Acad Dermatol 2015;73:37-49.A) 